\title{
Dinâmica da Floresta do Parque Estadual do Turvo com Índices de Vegetação
}

\author{
Paulo Afonso Rosa ${ }^{1}$, Fábio Marcelo Breunig ${ }^{1}$, \\ Rafaelo Balbinot ${ }^{1}$, Lênio Soares Galvão ${ }^{2}$ \\ ${ }^{1}$ Departamento de Engenharia Florestal - DEF, Universidade Federal de Santa Maria - UFSM, \\ Frederico Westphalen/RS, Brasil \\ ${ }^{2}$ Divisão de Sensoriamento Remoto, Instituto Nacional de Pesquisas Espaciais - INPE, São José dos Campos/SP, Brasil
}

\section{RESUMO}

Este trabalho analisou a relação entre índices de vegetação e precipitação, no Parque Estadual do Turvo (PET), localizado no noroeste do Estado do Rio Grande do Sul (RS). O Normalized Difference Vegetation Index (NDVI) e o Enhanced Vegetation Index (EVI) foram determinados a partir de imagens do Moderate Resolution Imaging Spectroradiometer (MODIS/Terra). Dados de precipitação do Tropical Rainfall Measuring Mission (TRMM) permitiram o cálculo do índice de estiagem. Foram identificados nove meses com precipitações abaixo da média, 92 meses com precipitações normais e 50 meses com valores acima da média. Os ciclos anuais de NDVI e EVI não apresentaram relação conclusiva com a precipitação anormal, mostrando que a floresta apresenta resiliência para suportar pequenos períodos de seca. O NDVI apresentou maior estabilidade no verão e no inverno.

Palavras-chave: sensoriamento remoto, séries temporais, chuva, resiliência.

\section{Dynamics of the Forest from the 'Parque Estadual do Turvo' with Vegetation Indices}

\begin{abstract}
In this study, we evaluated the relation between vegetation indices and rainfall in the 'Parque Estadual do Turvo' (PET), located in the northwest of Rio Grande do Sul (RS) state. The Normalized Difference Vegetation Index (NDVI) and Enhanced Vegetation Index (EVI) were determined from images of the Moderate Resolution Imaging Spectroradiometer (MODIS/ Terra). Rainfall data from the Tropical Rainfall Measuring Mission (TRMM) allowed the calculation of the dry spell index. Data analysis showed that nine months presented rainfall below the average; 92 months had rainfall close to the average; and 50 months presented rainfall above the average. The annual cycles of NDVI and EVI did not present conclusive correlation with the abnormal rainfall data, showing that the forest is resilient to short dry periods. The NDVI showed more stability in the summer and winter.
\end{abstract}

Keywords: remote sensing, time series, rain, resilience. 


\section{INTRODUÇÃO E OBJETIVOS}

A determinação da resposta fisiológica das plantas frente a distúrbios, como a variabilidade da temperatura do ar e a precipitação, é um desafio para a modelagem do estoque de carbono (Booth et al., 2012; Huntingford et al., 2013). A avaliação da dinâmica florestal com imagens orbitais e suborbitais pode fornecer indicadores das variações das características do dossel e permitir avaliar sua relação com a precipitação. A determinação da resiliência de uma floresta, que se refere à capacidade de um sistema para restabelecer seu equilíbrio após este ter sido rompido por um distúrbio (Gunderson, 2000), é um desafio para a correta modelagem de cenários frente às mudanças ambientais (Phillips et al., 2009). As variações em uma população vegetal ocorrem, por vezes, sazonalmente e com determinada periodicidade, em função de fatores bióticos e abióticos. Tais variações podem ainda ocorrer individualmente ou em nível de comunidade e população, havendo, assim, certa mudança fenológica sobre as características vegetais (Zhang et al., 2003). Mudanças sazonais da disponibilidade dos recursos hídricos ou variações na precipitação potencializam ou limitam o crescimento da vegetação e alteram a taxa de acumulação de biomassa e a taxa de senescência (Lee et al., 2002). As alterações em nível de dossel se manifestam através de parâmetros biofísicos, como o Îndice de Área Foliar (IAF), que podem ocasionar mudanças significativas no Fator de Reflectância Bidirecional (FRB), observado por sensores remotos imageadores.

A observação das variações fenológicas da vegetação através de índices de vegetação, calculados a partir de valores de FRB, obtidos de imagens orbitais de alta resolução temporal, é usada para a análise integrada dos componentes de uma paisagem e para o entendimento e a predição de suas mudanças (Vibrans \& Schramm, 2009). Várias técnicas e produtos foram desenvolvidos para analisar a mudança na estrutura da vegetação a partir de fatores ambientais e antrópicos. Uma das fontes de dados amplamente utilizadas para estudos em escala regional ou global refere-se ao sensor Moderate Resolution Imaging Spectroradiometer (MODIS), a bordo dos satélites Terra e Aqua. O sensor MODIS foi projetado com 36 bandas, com resolução espacial variando de 250 a 1000 metros e com cobertura global quase diária (Justice et al., 2002). A utilização desses dados permite uma análise multitemporal adequada, que pode auxiliar e otimizar as inspeções de campo.

Um dos produtos MODIS disponibilizados para a comunidade de sensoriamento remoto inclui a determinação dos índices de vegetação Normalized Difference Vegetation Index (NDVI) e Enhanced Vegetation Index (EVI). Índices de vegetação são formados pela combinação de bandas espectrais, que podem ser adicionadas, subtraídas, divididas ou multiplicadas, de forma a produzir um valor único que indique a quantidade ou o vigor de vegetação (Santos et al., 2010), reduzindo os impactos dos fatores externos (sombreamento, relevo, atmosfera, etc.). Índices como NDVI e EVI exploram as diferenças na resposta espectral da vegetação nas faixas do azul, do vermelho e do infravermelho próximo, para a sua detecção nas imagens (Baret et al., 1989). Esses índices de vegetação podem ser utilizados empiricamente para estimar a quantidade de biomassa e caracterizar o estado fenológico da vegetação, uma vez que a produção primária de uma comunidade vegetal mostra estreita relação com a energia (irradiância solar) absorvida por ela (Baret \& Guyot, 1991; Ponzoni, 2001).

ONDVIéumíndice devegetação maistradicional, mas tende a saturar em florestas, dependendo do índice de área foliar (IAF) (Rouse et al., 1973; Silva et al., 2009). O EVI foi desenvolvido para amenizar esse problema, melhorando a sensibilidade em regiões com maiores densidades de biomassa, minimizando a influência do substrato para dosséis esparsos e auxiliando na redução de influências atmosféricas (Huete et al., 2002; Justice et al., 1998). Em florestas subtropicais, os valores de NDVI e EVI podem variar sazonalmente devido às modificações nos parâmetros bioquímicos e biofísicos da vegetação, ou na fenologia da vegetação. Em princípio, NDVI e EVI são mais sensíveis aos parâmetros biofísicos que refletem indiretamente a estrutura da vegetação (p.ex., IAF, biomassa).

A precipitação atmosférica é um dos principais fatores que determinam o estresse fisiológico da vegetação. O sistema sensor Tropical Rainfall Measuring Mission (TRMM) possibilita 
o acompanhamento, a previsão e a análise de precipitação. Durante mais de 14 anos, os sensores a bordo do satélite TRMM monitoram nuvens, precipitações, fluxo de calor, raios e outros aspectos do ciclo da água. O TRMM foi lançado em 1997 como um satélite experimental da National Aeronautics and Space Administration (NASA) e da Japan Aerospace Exploration Agency (JAXA). Em virtude dos bons resultados alcançados, seu status foi alterado para satélite operacional, gerando previsões atmosféricas (Passow, 2010).

O Parque Estadual do Turvo (PET), criado em 1947, é um dos poucos remanescentes de floresta subtropical da região noroeste do Rio Grande do Sul. Seus arredores foram intensamente desflorestados para a implementação da agropecuária (Leite, 2002). Por ser uma área florestal que abriga uma imensa riqueza biológica de espécies de flora nativa do Rio Grande do Sul, faz-se necessário um estudo sobre o comportamento da vegetação, quando submetida a diferentes níveis de precipitação, especialmente considerando-se cenários futuros com possíveis mudanças climáticas e maior ocorrência de eventos extremos.

O objetivo do presente trabalho é analisar a dinâmica espectro-temporal da floresta subtropical do PET, utilizando-se índices de vegetação (NDVI e EVI) do sensor MODIS/Terra e dados pluviométricos do TRMM, e discutir a relação entre os índices de vegetação e a precipitação.

\section{MATERIAL E MÉTODOS}

Para facilitar o entendimento dos materiais e métodos adotados no estudo, a Figura 1 ilustra as principais etapas do desenvolvimento do trabalho.

\section{1. Área de estudo}

Localizado no município de Derrubadas-RS, o PET tem cerca de 17.500 ha de área florestal (Figura 2). O PET constitui um dos últimos redutos representativos da vegetação original da mata pluvial do Alto Uruguai, que ocupava uma grande área das Bacias dos Rios Paraná e Uruguai (Brack et al., 1985). A temperatura média anual do município é de $19,1^{\circ} \mathrm{C}$ e a precipitação pluviométrica é de $1.800 \mathrm{~mm}$. O relevo do município apresenta-

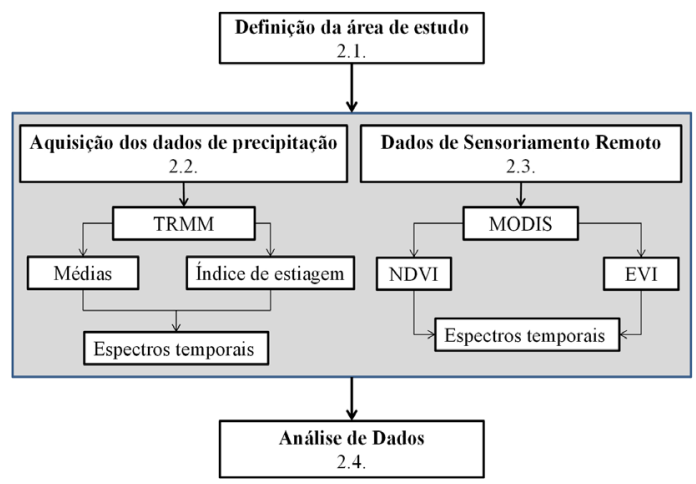

Figura 1. Fluxograma com as principais etapas do estudo.

Figure 1. Workflow of the main steps of the study.

se ondulado em aproximadamente $55 \%$ da área, acidentado em 40\% e plano em apenas 5\% (Rio Grande do Sul, 2005; Derrubadas, 2012). Em geral, o parque apresenta solo argiloso, pobre em quartzo, rico em ferro e manganês, com pouca profundidade média, drenagem rápida e susceptibilidade à erosão.

O PET é uma unidade de conservação de proteção integral. Foi criado primeiramente como sendo Reserva Florestal Estadual do Turvo, através do Decreto n. ${ }^{\circ}$ 2312, de 11 de março de 1947. Em 2 de outubro de 1954, pelo efeito da Lei n. ${ }^{\circ} 2440$, foi determinado que todas as matas de domínio do Estado e com mais de 250 ha fossem transformadas em Parques Estaduais. A então Reserva Estadual Florestal do Turvo passou à categoria de Parque Florestal Estadual do Turvo (Parque Florestal Estadual do Turvo, 2012). A área do PET é de $17.491,4$ ha, representando quase $50 \%$ da área total do município de Derrubadas e 0,065\% da área do Estado do Rio Grande do Sul.

A vegetação é predominantemente composta pela Floresta Estacional Decidual (Floresta Tropical Caducifólia) (IBGE, 2012), apresentando também áreas de banhado, lajeado e formações antrópicas (Parque Florestal Estadual do Turvo, 2012). No contexto nacional, o PET preserva uma significativa amostra da Floresta Estacional Decidual (IBGE, 1986). Essa área representa o último grande fragmento desse tipo de vegetação no Rio Grande do Sul, que, segundo Irgang (1980), estendia-se originalmente desde o Estado do Paraná. Por sua 


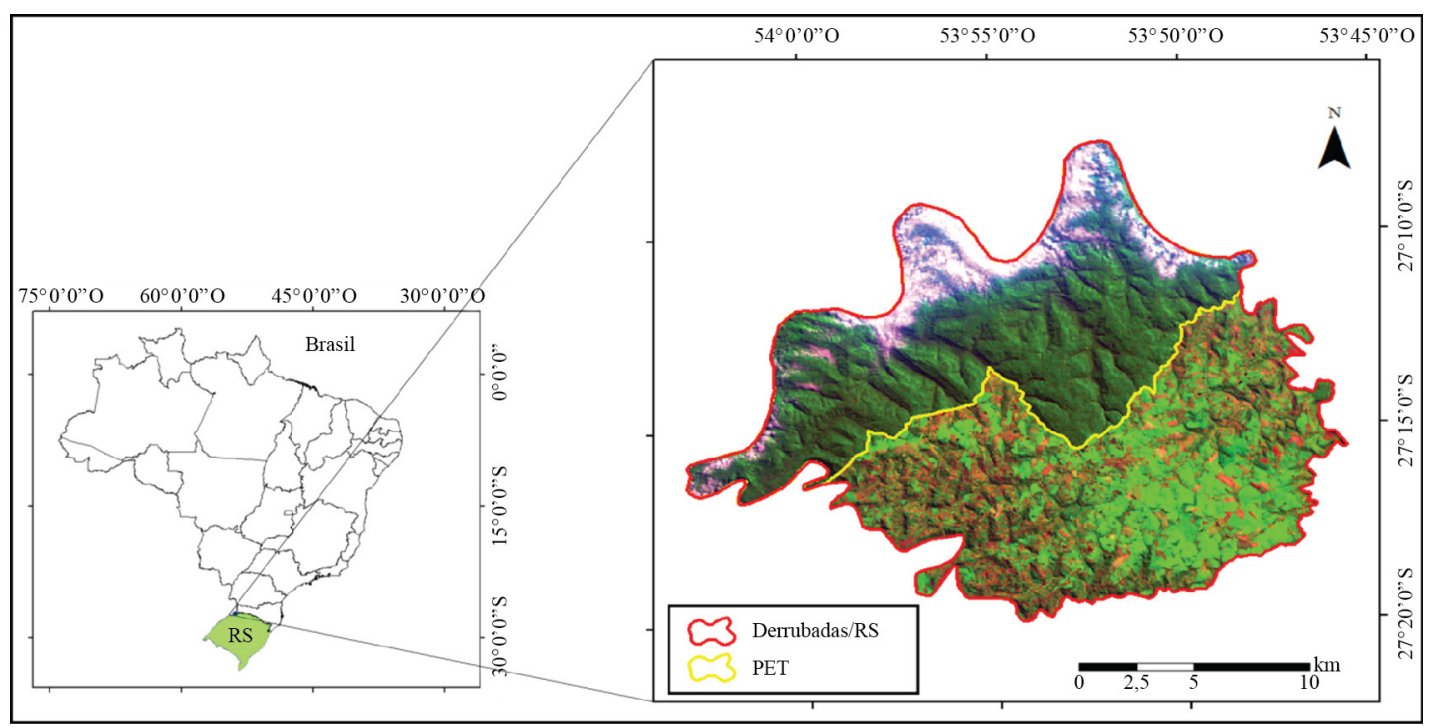

Figura 2. Localização do Parque Estadual do Turvo (PET), no noroeste do Estado do Rio Grande do Sul. A linha amarela (branca na impressão $P \& B$ ) na figura da direita indica os limites do PET. Fonte: Imagem Landsat 5 TM de novembro de 2010.

Figure 2. Location of the Parque Estadual do Turvo (PET), in the northwest portion of the Rio Grande do Sul State. At the right side of the figure, the yellow line (white in the grayscale print) indicates the limits of the PET. Source: Landsat 5 TM image from November 2010.

relevância, o PET é reconhecido como uma área de extrema importância para a conservação da biodiversidade.

\subsection{Aquisição dos dados de precipitação}

Os dados mensais de precipitação da área de estudo foram obtidos através do satélite TRMM, provenientes do repositório da NASA (FTP disc2. nascom.nasa.gov). Os dados de precipitação TRMM são gerados a partir da combinação dos produtos 1B01 (gerado a partir do TRMM Visible and Infrared Scanner - VIRS), 2A12 (TRMM Microwave Imager - TMI) e 3B31 (monthly TMI/TRMM Combined Instrument - TCI) com os dados de satélites, como GMS, GOES-E, GOES-W, Meteosat-7, Meteosat-5 e NOAA-12. Como resultado da combinação, é gerado o produto 3B42-v7 (3-hourly TRMM and Other Rainfall Estimate - version 7 - TRMM-Adjusted Merged-Infrared Precipitation). Os dados TRMM foram obtidos no nível 3B42-v7 e processados através de um algoritmo do GrADS (Grid Analysis and Display System) (COLA, 2010) e IDL-ENVI (ITT VIS, 2008). Os dados foram rearranjados para representar a precipitação mensal acumulada.
Os períodos chuvosos e de estiagem foram determinados pelo Índice de Estiagem (IE). A definição de período de estiagem segue a metodologia de Castro (1996), que define estiagem como o período com precipitação mensal menor do que $40 \%$ da média histórica do mês. Já a estação chuvosa foi considerada como sendo o período com precipitação mensal maior do que $150 \%$ da média histórica. Assim, foi determinado o IE a partir das médias históricas mensais, sendo este o quociente entre a precipitação mensal e a média histórica do mês.

\subsection{Dados de sensoriamento remoto - NDVI e EVI}

Para análise da região de estudo, foram utilizadas as imagens MODIS/Terra, produto MOD13Q1 (Vegetation Indices 16-Day L3 Global $250 \mathrm{~m}$ ), que contêm os índices de vegetação EVI e NDVI, obtidos do servidor FTP do United States Geological Survey (USGS, e4ft101.cr.usgs.gov). Os dados MODIS foram pré-processados no algoritmo MODIS Reprojection Tool (MRT) (Dwyer \& Schmidt, 2006), a fim de transformar os dados de localização geográfica em coordenadas planares (zona UTM 22, WGS-84). As 
séries de índices de vegetação foram analisadas no aplicativo ENVI (ITT VIS, 2008).

O NDVI e o EVI são calculados através das Equações 1 e 2, respectivamente. Os cálculos foram realizados utilizando-se ferramentas de álgebra implementadas no aplicativo ENVI 4.5 (ITT VIS, 2008).

$$
\begin{aligned}
& N D V I=\frac{(\rho I V P-\rho V)}{(\rho I V P+\rho V)} \\
& E V I=\frac{(\rho I V P-\rho V)}{\left(\rho I V P+C_{1} \cdot \rho V-C_{2} \cdot \rho A+L\right)}
\end{aligned}
$$

em que: $\rho \mathrm{IVP}, \rho \mathrm{V}$ e $\rho \mathrm{A}=$ valores de reflectância das bandas do infravermelho próximo, vermelho e do azul, respectivamente; $L=$ fator de ajuste do solo, adotado no produto MOD13Q1 como igual a 1, valor considerado adequado pela equipe do MODIS para o monitoramento global dos biomas (Huete et al., 1992); $C 1(6)$ e $C 2(7,5)=$ coeficientes de ajuste para efeito de aerossóis da atmosfera; $\mathrm{G}(2,5)=$ fator de ganho (Miura et al., 2001).

Na medida em que o produto MOD13Q1 referese a uma composição de 16 dias, foram adquiridas aproximadamente duas imagens mensais para o período de fevereiro de 2000 a agosto de 2012, totalizando 151 meses. Posteriormente, foi criada uma máscara para extração da área correspondente ao PET. Para a determinação das médias de NDVI e EVI, foi feita uma amostragem computacional aleatória na região de estudo, com um percentual de amostragem de $10 \%$ da área do PET. Feito isso, foram computadas as estatísticas para cada índice de vegetação. Para a análise dos dados NDVI e EVI, foram obtidos os valores das duas imagens de cada mês e utilizado um valor médio mensal, uma vez que os dados de precipitação também são mensais. Todos os processamentos de dados foram executados no aplicativo ENVI versão 4.5 (ITT VIS, 2008). A análise dos dados foi realizada através de gráficos e tabelas.

\subsection{Análise de dados}

O NDVI e o EVI foram avaliados a partir de série de imagens MODIS de 2000 a 2012. A partir dos dados dos índices de vegetação, foram elaborados gráficos ilustrando as variações intra e interanuais. Com base nos gráficos, foi possível identificar os períodos em que a vegetação apresentou os maiores e menores valores de NDVI e EVI. As variações interanuais viabilizaram a avaliação da dinâmica da floresta do PET ao longo dos 12 anos estudados. A variação intra-anual permitiu identificar as características de cada ciclo anual da floresta, como a duração e a magnitude dos valores dos índices de vegetação em cada estação do ano. Além disso, permitiu uma avaliação comparativa entre os índices de vegetação.

Os dados de precipitação foram estudados considerando-se a precipitação diária acumulada e a média da precipitação do período para cada mês. Os dados foram representados graficamente e avaliados de forma comparativa. A precipitação estimada foi utilizada para calcular o IE. As estimativas de precipitação e do IE foram comparadas visualmente com os dados de NDVI e EVI, numa perspectiva intra e interanual.

Para gerar dados quantitativos de covariância entre os dados de precipitação e IE, e os dados derivados das medidas de reflectância (NDVI e EVI), foi utilizada a correlação cruzada. Considerando-se que a resposta espectral da floresta às variações de precipitação não é imediata (efeito ocorre com um atraso aproximado de 30 a 90 dias), foram avaliados atrasos de até quatro meses.

\section{RESULTADOS E DISCUSSÕES}

\subsection{Padrão temporal da precipitação com dados TRMM}

As variações das estimativas de precipitação mensal média acumulada entre 2000 e 2012 são apresentadas na Figura 3. Observa-se que as chuvas são bem distribuídas durante o ano, com mais de $100 \mathrm{~mm}$ mensais médios. A maior média de precipitação mensal ocorre no mês de outubro, seguida pelo mês de abril. Já os meses com menor índice mensal médio de precipitação são agosto $\mathrm{e}$ março. De acordo com os dados pluviométricos normais, elaborados pela Secretaria Estadual do Meio Ambiente (Rio Grande do Sul, 2005), podese observar um comportamento pluviométrico semelhante, ou seja, com precipitações bem distribuídas ao longo do ano, tendo seus maiores índices registrados nos meses de abril, maio, junho 
e outubro, e seus menores índices registrados nos meses de fevereiro, julho, agosto e dezembro. A Figura 4 apresenta as estimativas dos ciclos anuais de precipitação mensal acumulada, para o período entre 2000 e 2012. Observa-se que, em todos os anos analisados, há uma grande variação pluviométrica. Em alguns meses, ocorrem altas precipitações (aproximadamente $500 \mathrm{~mm}$ ) e, em outros meses, ocorrem baixas precipitações (inferiores a $50 \mathrm{~mm}$ ). A inspeção da Figura 4 mostra que alguns anos são marcados por fortes quedas das estimativas de precipitação no período do inverno (por exemplo, o inverno de 1999, 2007 e 2012). Esse resultado fica evidente nas barras do desvio padrão da Figura 3. Os meses com maior variabilidade interanual são dezembro, abril, fevereiro e agosto $( \pm 116,6 ; \pm 105,7$; $\pm 104,0$ e $\pm 100,3 \mathrm{~mm}$, respectivamente). Dada essa variabilidade, esperam-se algumas variações nos índices de vegetação nos meses seguintes aos meses atípicos. Da mesma forma, ocorrem alguns anos com forte aumento nas estimativas de precipitação no verão de 1998, 2003, 2004, 2008 e 2010. Nesses anos, no período do verão (ou meses próximos), a precipitação ficou acima de $400 \mathrm{~mm}$ mensais.
Levando-se em conta a metodologia proposta por Castro (1996), verificou-se que, para apenas nove meses, a precipitação estimada esteve abaixo da esperada (IE $<0,4$; abaixo da linha vermelha na Figura 5). Para outros 50 meses, a precipitação esteve acima da média (IE > 1,5; acima da linha azul na Figura 5). Finalmente, em 92 meses, a precipitação ocorreu normalmente ou dentro do esperado $(0,4<$ IE $<1,5$; entre as linhas vermelha e azul na Figura 5). Esses dados são consistentes com a classificação do IBGE (2012) para essa área como zona subtropical sem período seco, porém com inverno frio, que determina o repouso fisiológico e a queda parcial da folhagem. Assim, o caráter decidual da florestal do PET parece estar relacionado à temperatura.

Segundo dados do Centro de Previsão de Tempo e Estudos Climáticos (CPTEC, 2013), ocorreram eventos de fraca intensidade de El Niño entre os anos de 2004 e 2005 e 2006 e 2007, intensidade moderada entre 2009 e 2010, e fraco em 2012. (CPTEC, 2013). Comparando-se esses resultados com os dados da Figura 5, verifica-se que ocorreram três períodos de estiagem entre 2004 e 2005, dois entre 2006 e 2007, dois entre 2009 e 2010, e dois em 2012. Isso mostra

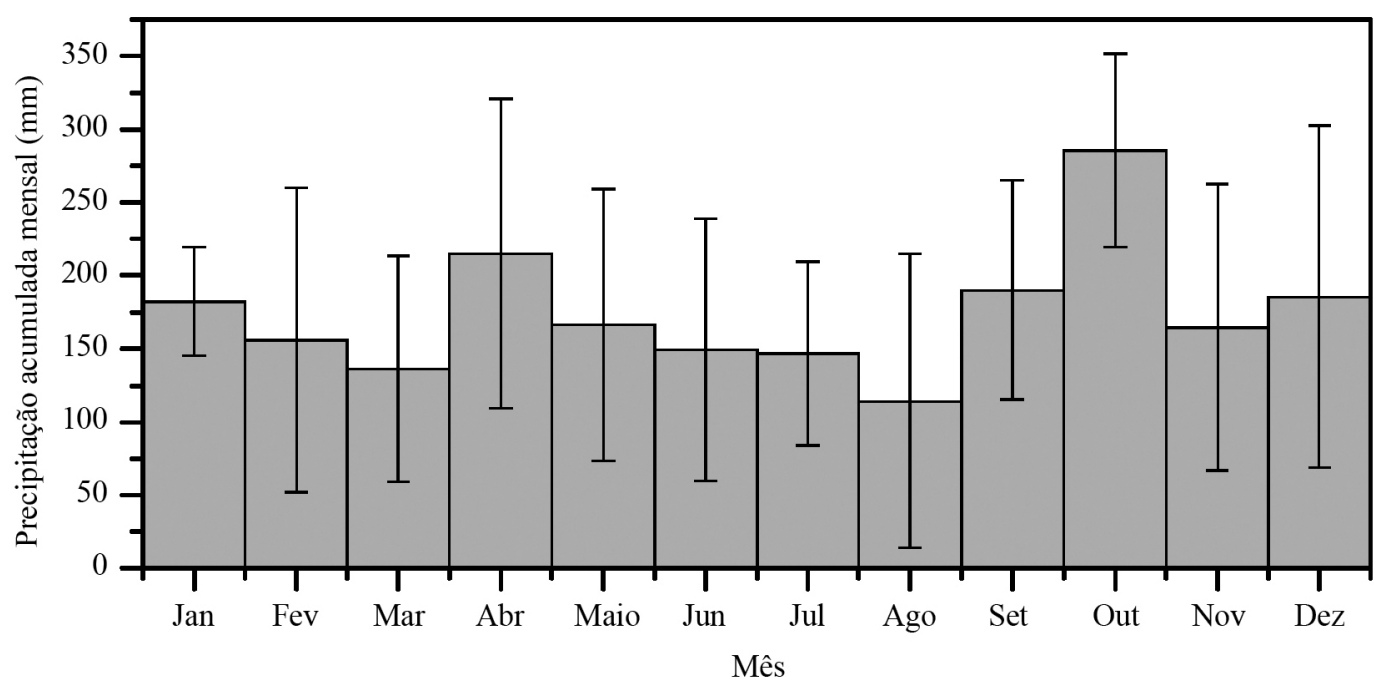

Figura 3. Estimativa da precipitação mensal acumulada média, gerada pelo satélite TRMM (TRMM Produto 3B42-V7). Os dados representam a média de 12 anos de medidas (2000 a 2012), calculados sobre a área do PET. As barras de erro são mostradas a cada mês. A validação dos dados TRMM é apresentada por Franchito et al. (2009). Figure 3. Average monthly rainfall estimates generated from the TRMM satellite (TRMM Product 3B42-V7). The data represent a 12-year period mean (2000 to 2012), calculated over the PET. The error bars are shown per month. TRMM data validation is presented in Franchito et al. (2009). 


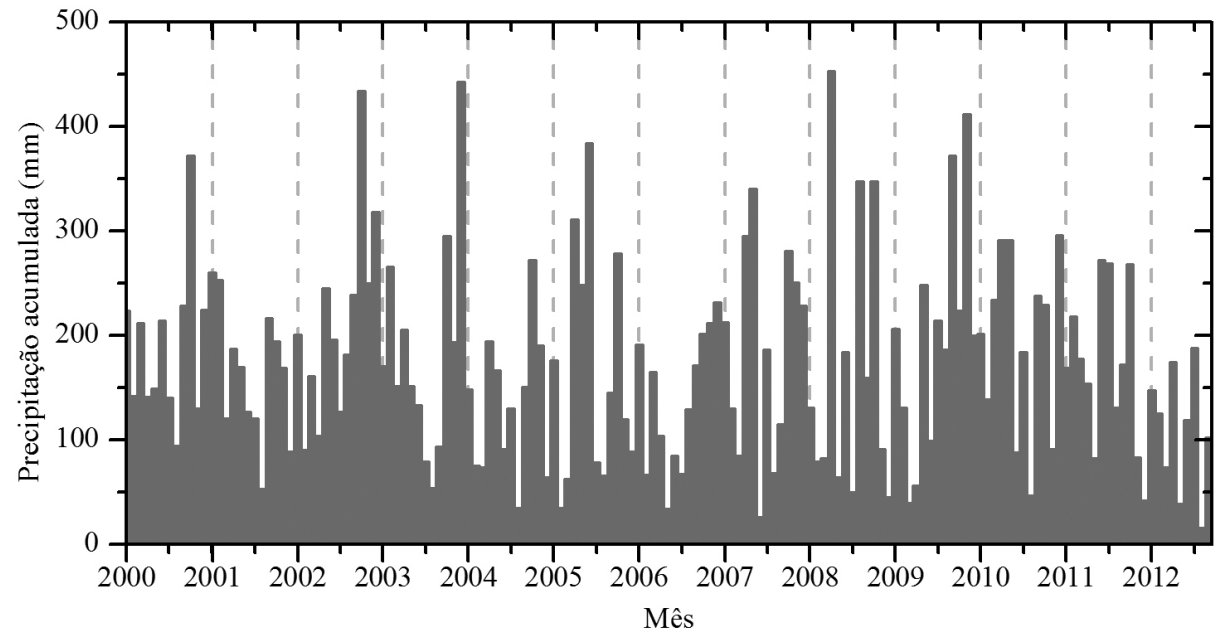

Figura 4. Estimativa dos ciclos anuais de precipitação mensal, gerada pelo satélite TRMM (TRMM Produto 3B42-V7). Os dados representam a média de 12 anos de medidas (2000 a 2012), calculados sobre a área do PET.

Figure 4. Annual cycles of monthly estimates of precipitation generated from the TRMM satellite (TRMM Product 3B42-V7). The data represent a 12-year period mean (2000 to 2012), calculated over the PET.

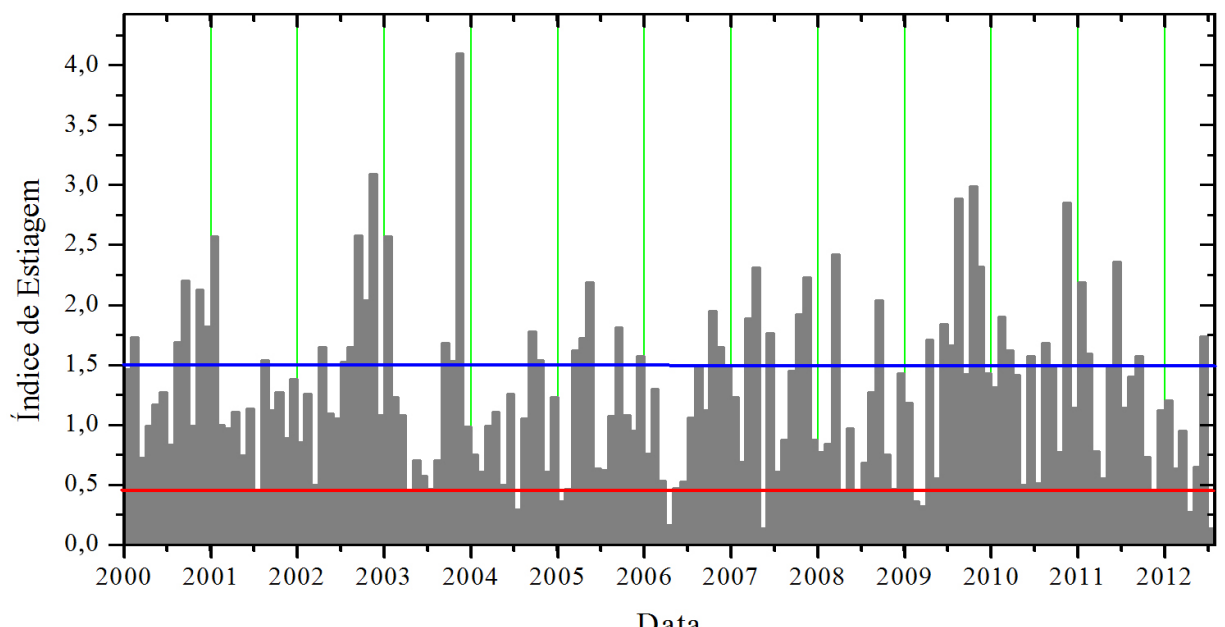

Figura 5. Índice de estiagem no PET para o período de 2000 a 2012, baseado nas estimativas de precipitação do TRMM. As linhas vermelha e azul indicam o limiar de chuva abaixo (seca) e acima do índice normal, respectivamente. Figure 5. Dry spell Index (IE) in the PET for the period from 2000 to 2012, based on TRMM rainfall estimates. The red and blue lines indicate the thresholds for rainfall below (dry spell) and above the average or expected precipitation, respectively.

que as maiores estiagens coincidem com os eventos de El Niño nesta área de estudo no Sul do Brasil.

\subsection{Padrão temporal dos índices de vegetação}

Os índices de vegetação NDVI e EVI são mais sensíveis às variações biofísicas do dossel, como a redução do Índice de Área Foliar (IAF). Pelo fato da floresta do PET apresentar um caráter decidual, em que mais de $50 \%$ dos indivíduos apresentam uma perda de folhas em períodos desfavoráveis, são gerados ciclos anuais típicos para o NDVI e o EVI, refletindo esse aspecto (Figura 6). Apesar dos ciclos anuais repetitivos, observaram-se variações intra e interanuais tanto para o NDVI quanto para o EVI. Analisando-se todo o período de 2000 a 2012, verificou-se uma gradual redução dos valores de NDVI e EVI do início para o final da série temporal, 
com variações nos valores máximos e mínimos dos índices. As variações interanuais podem estar associadas às diferenças nos índices de precipitação, conforme visto no ano de 2009 (Figura 5), aos efeitos atmosféricos, à geometria de iluminação e de aquisição dos dados, ao sombreamento interno do dossel e ao dia selecionado para a geração da composição 16-dias (produto MOD13Q1), entre outros fatores. Segundo Atkinson et al. (2011), o declínio gradual observado na série temporal dos índices de vegetação do MODIS na Amazônia, de 1999 para 2011, pode ter como causa fatores atmosféricos ou ruído instrumental. Os autores destacam a necessidade de estudos adicionais para compreender tal fenômeno.

Comparativamente, o EVI mostrou variações mais acentuadas e foi mais sensível às oscilações sazonais da resposta espectral da vegetação do que o NDVI. Esse fato pode ser resultante da maior sensibilidade do EVI às variações de reflectância da banda do infravermelho próximo (Galvão et al.,
2013). No mesmo sentido, o NDVI foi mais estável dentro das estações, fato evidenciado pelos platôs.

\subsection{Relação dos índices de vegetação com a estimativa de precipitação}

Nas Figuras 7 e 8, são apresentados os comportamentos das médias dos valores de precipitação e dos valores de NDVI e EVI, respectivamente. Há uma semelhança entre o comportamento dos índices de vegetação e a precipitação. $\mathrm{O}$ aumento da precipitação parece resultar em maiores valores de índices de vegetação. Contudo, isso ocorre porque os meses com maior precipitação coincidem com a primavera chuvosa e com a tendência de aumento da temperatura. Sua redução produz menores valores de NDVI e EVI, coincidindo com o período de inverno. Com a redução da precipitação no período do inverno, quando o frio é mais intenso, a floresta do PET perde folhas, o que causa diminuição nos valores dos

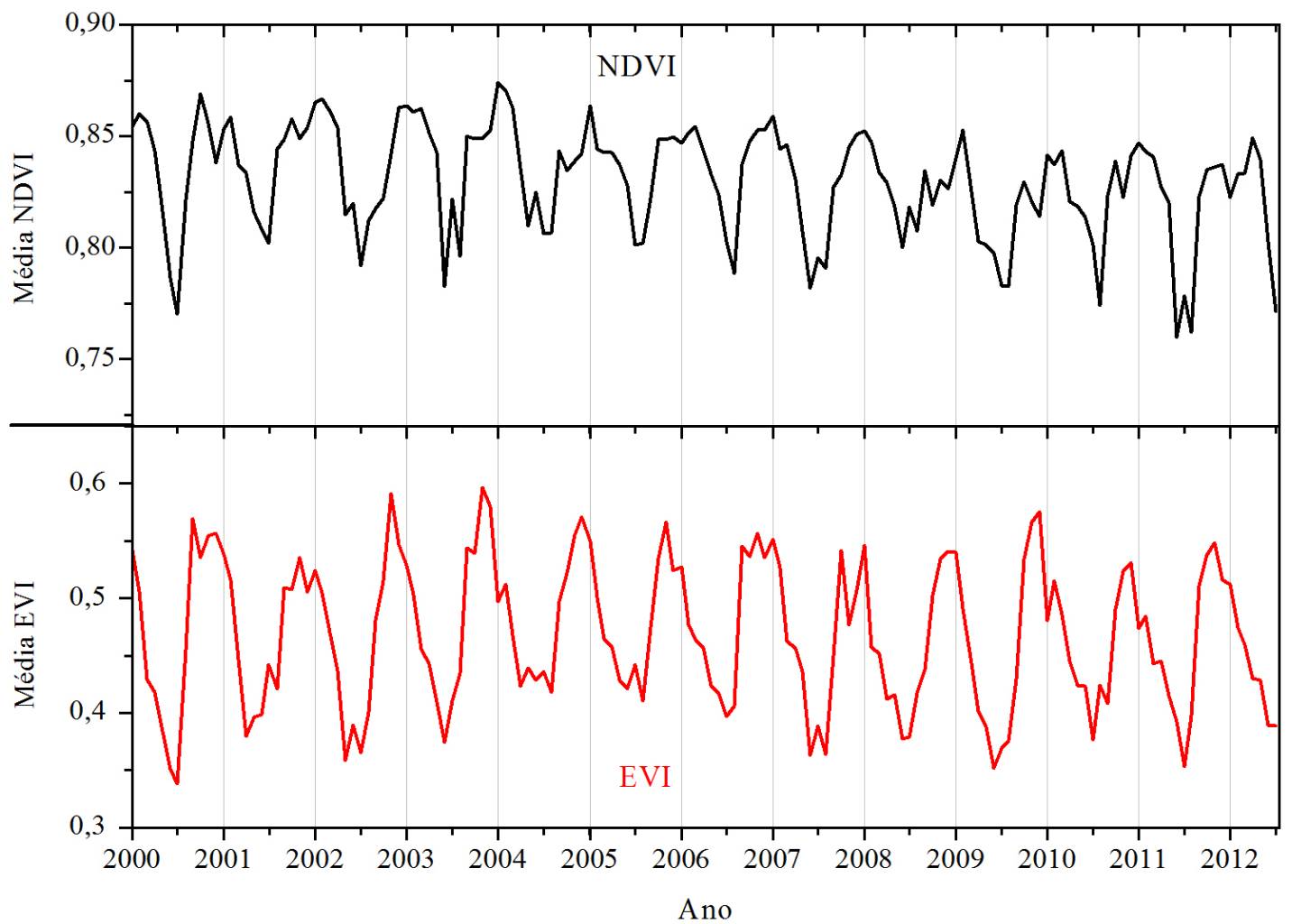

Figura 6. Ciclos anuais de NDVI e EVI, calculados a partir das composições 16 dias do MODIS, usando 10\% dos pixels selecionados aleatoriamente no PET.

Figure 6. Annual cycles of NDVI and EVI, calculated from the 16-days compositing MODIS product, using $10 \%$ of the pixels selected randomly over the PET. 


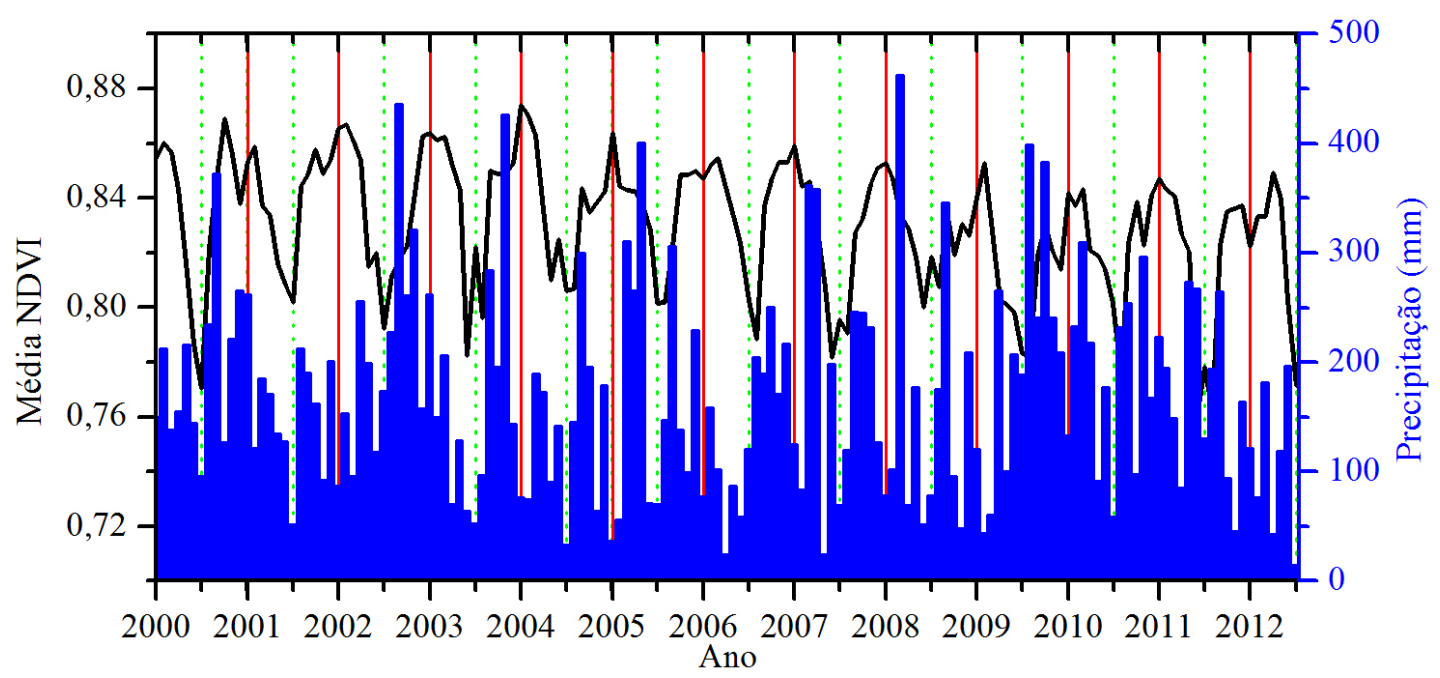

Figura 7. Variação do NDVI em função das estimativas de precipitação do satélite TRMM.

Figure 7. Variations of NDVI as a function of the rainfall TRMM estimates.

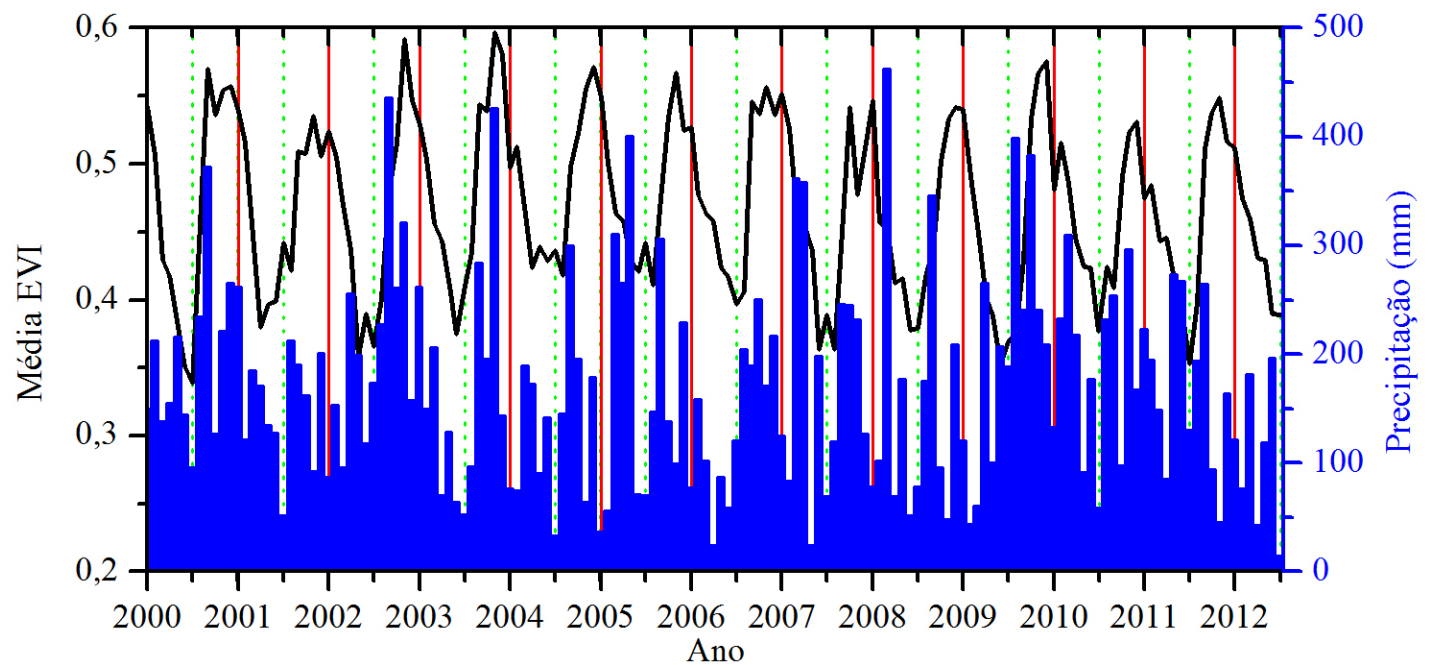

Figura 8. Variação do EVI em função das estimativas de precipitação do satélite TRMM.

Figure 8. Variations of EVI as a function of rainfall estimates from the TRMM satellite.

índices de vegetação. Assim, os resultados indicam que os meses com menor precipitação podem intensificar a perda de folhas induzida pelo frio.

Analisando-se as Figuras 7 e 8, não é possível estabelecer uma relação direta entre os picos de precipitação e o aumento posterior dos índices de vegetação. Assim, acredita-se que a influência da precipitação é mais acentuada por variações prolongadas de excesso ou escassez de precipitações. Esse fato pode ser entendido quando se considera a relação entre índices de vegetação e o índice de estiagem (Figura 9).

A avaliação das variações do IE bimestralmente mostra suas significativas oscilações ao longo do período estudado (Tabela 1). Observa-se que, de um mês para outro, os valores do IE aumentam e retornam à normalidade, ou seja, em um mês ocorre estiagem e, no mês seguinte, a precipitação ocorre normalmente. Assim, são bimestres marcados por fortes variações, em que se esperariam variações dos índices de vegetação no final do bimestre, 


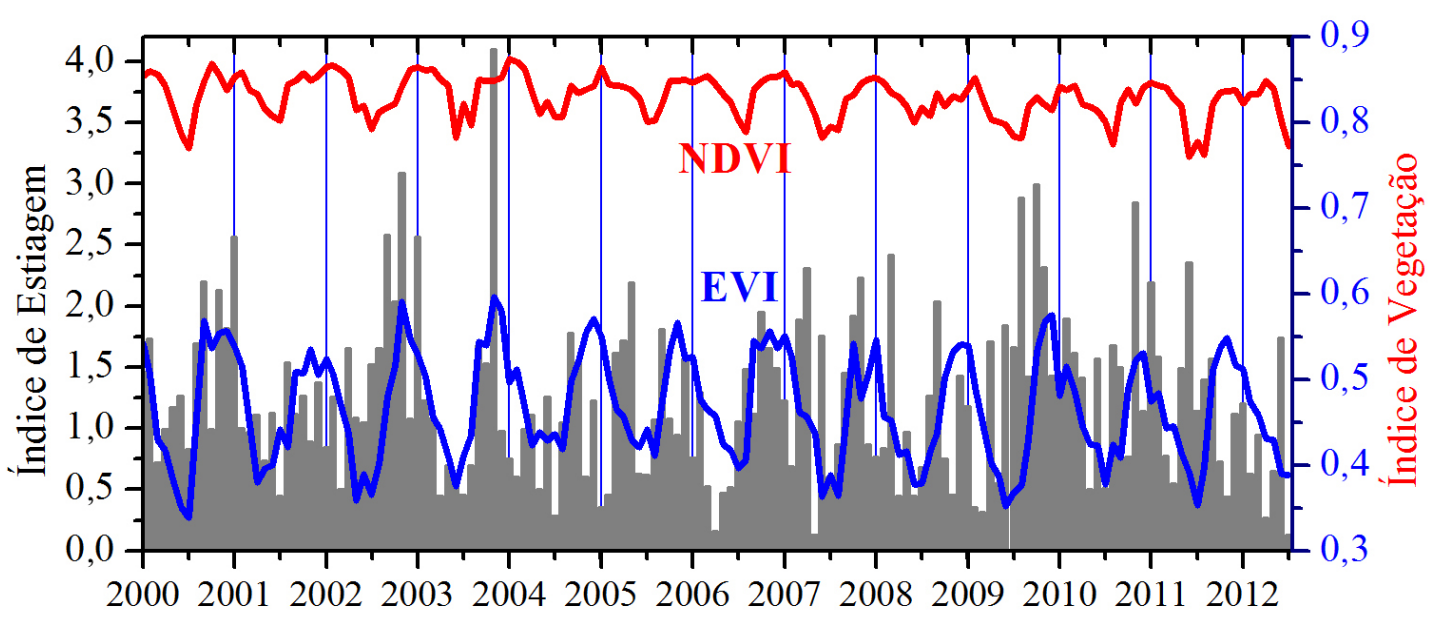

\section{Data}

Figura 9. Variações do NDVI e do EVI em função do índice de estiagem (IE).

Figure 9. Variations of NDVI and EVI as a function of the dry spell index.

Tabela 1. Bimestres que apresentaram mudanças significativas nos IE e as variações associadas do NDVI e do EVI. Table 1. Bimesters that presented significant changes in IE (dry spell index) and the associated variations of NDVI and EVI.

\begin{tabular}{|cccc|}
\hline Mês-Ano & IE & NDVI & EVI \\
\hline ago./04-set./04 & $0,29-1,05$ & $0,81-0,81$ & $0,42-0,50$ \\
\hline fev./05-mar./05 & $0,36-0,46$ & $0,86-0,84$ & $0,55-0,50$ \\
\hline maio/06-jun./06 & $0,16-0,47$ & $0,84-0,83$ & $0,46-0,42$ \\
\hline jun./07-jul./07 & $0,13-1,76$ & $0,81-0,78$ & $0,44-0,36$ \\
\hline mar./09-abr./09 & $0,36-0,32$ & $0,85-0,83$ & $0,49-0,45$ \\
\hline abr./09-maio/09 & $0,32-1,71$ & $0,83-0,80$ & $0,45-0,40$ \\
\hline maio/12-jun./12 & $0,27-0,65$ & $0,85-0,83$ & $0,44-0,44$ \\
\hline ago./12 & 0,13 & 0,77 & 0,39 \\
\hline
\end{tabular}

especialmente se a resiliência da floresta fosse baixa a secas. Analisando-se a Tabela 1, juntamente com a Figura 9, observa-se que, em alguns momentos, há uma discordância entre os IE e os índices de vegetação, ou seja, mesmo com o aumento dos IE, os índices de vegetação tendem a ter um comportamento contrário. Há uma mudança no IE, porém os índices de vegetação não acompanham essa mudança. $O$ fato é que tais mudanças são por períodos muito curtos (um mês) e a floresta ainda tem recursos (água) para seus processos fisiológicos. Somente uma seca prolongada poderia gerar um déficit hídrico que se manifestasse através da resposta espectral das plantas. A vegetação tende a responder menos a curtos períodos extremos do que a longos períodos de seca ou de chuva em abundância. Isto se chama resiliência hídrica da floresta, ou seja, pode-se, a partir desses dados, afirmar que, durante o período de estudo, a floresta teve capacidade de suportar curtos períodos de estiagem sem sofrer alterações significativas ou visíveis no dossel, considerando-se a metodologia deste trabalho.

Entre as técnicas disponíveis para comparar séries temporais, a correlação cruzada é uma das mais utilizadas. Essa avaliação quantitativa, baseada na correlação cruzada mostrou uma maior correlação entre o EVI $(0,188)$ e a precipitação acumulada do que do NDVI $(0,113)$ com a precipitação. Para ambos os índices, os melhores ajustes foram encontrados considerando-se um mês de atraso (significância de 0,05). Levando-se em conta as relações entre IE e o EVI e NDVI, as melhores correlações foram encontradas para o EVI $(0,301)$ com um mês de 
atraso e o NDVI $(0,238)$ com três meses de atraso. Apesar de essas correlações terem sido significativas, os ajustes foram muito baixos, mostrando que a floresta do PET apresenta uma resiliência a pequenos períodos de estiagem.

\section{CONCLUSÕES}

No período estudado, apenas nove meses tiveram precipitação abaixo da média, coincidindo com os eventos de El Niño, enquanto 92 meses tiveram índices pluviométricos normais e 50 meses tiveram precipitações pluviométricas acima do esperado. Com base nos 12 anos avaliados, através da comparação dos dados de precipitação do TRMM e índices de vegetação, não foi identificada uma dependência clara da floresta em relação às estimativas de precipitação. $\mathrm{O}$ padrão pluviométrico não afetou as características do dossel em termos de reflectância dentro do período analisado. A floresta do PET apresentou resiliência suficiente para suportar esses pequenos períodos de estiagem. O padrão de precipitação pluviométrica não oscilou de forma a gerar alterações no dossel dessa floresta identificáveis pelos índices NDVI e EVI. Novos estudos serão desenvolvidos com medidas in loco do IAF e dados de temperatura.

O NDVI e o EVI permitiram avaliar a dinâmica intra e interanual da floresta do PET, apresentando uma diminuição dos seus valores de 2000 a 2012. Os índices de vegetação avaliados apresentaram pequenas variações intra-anuais. O NDVI apresentou maior estabilidade nos períodos de verão e inverno, quando comparado ao EVI, considerando-se as oscilações sazonais intra-anuais.

Os resultados apresentados requerem novos estudos que avaliem a dependência da floresta do PET às variações de temperatura e com maior detalhamento da ocorrência de fenômenos como El Niño e La Niña, e a ocorrência de frentes frias.

\section{AGRADECIMENTOS}

Os autores agradecem à Fundação de Amparo à Pesquisa do Estado do Rio Grande do Sul (FAPERGS), pelo apoio financeiro (ARD - processo n. ${ }^{\circ}$ 12/0383-5 de 2012), à Universidade Federal de Santa Maria (UFSM - projeto n. ${ }^{\circ} 031263$ e projeto n. ${ }^{\circ}$ 030745) e à Fundação de Amparo à Pesquisa do Estado de São Paulo (projeto 2013/03908-3).

\section{STATUS DA SUBMISSÃO}

Recebido: 01/04/2013

Aceito: 17/08/2013

Publicado: 31/12/2013

\section{AUTOR(ES) PARA CORRESPONDÊNCIA}

\section{Fábio Marcelo Breunig}

Departamento de Engenharia Florestal - DEF,

Universidade Federal de Santa Maria - UFSM,

Campus do CESNORS, CEP 98400-000,

Frederico Westphalen, RS, Brasil

e-mail: breunig@ufsm.br

\section{REFERENNCIAS}

Atkinson PM, Dash J, Jeganathan C. Amazon vegetation greenness as measured by satellite sensors over the last decade. Geophysical Research Letters 2011; 38(19). http://dx.doi.org/10.1029/2011GL049118

Baret F, Guyot G. Potential and Limits of Vegetation Índices for LAI e APAR Assessment. Remote Sensing of Environment 1991; 3: 161-173. http://dx.doi. org/10.1016/0034-4257(91)90009-U

Baret F, Guyot G, Major D. TSAVI: a vegetation index which minimizes soil brightness effects on LAI or APAR estimation. In: Proceedings of the Canadian symposium on remote sensing; 1989; Vancouver. Ottawa: Canadian Remote Sensing Society; 1989.

Booth BBB, Jones CD, Collins M, Totterdell IJ, Cox PM, Sitch S, et al. High sensitivity of future global warming to land carbon cycle processes. Environmental Research Letters 2012; 7(2): 024002. http://dx.doi. org/10.1088/1748-9326/7/2/024002

Brack P, Bueno RM, Falkemberg DB, Paiva MRC, Sobral M, Stehmann JR. Levantamento florístico do Parque Estadual do Turvo, Tenente Portela, Rio Grande do Sul, Brasil. Roessléria 1985; 7: 69-94.

Castro ALC. Desastres naturais. Brasília: Ministério do Planejamento e Orçamento; 1996.

Center for Ocean-Land-Atmosphere Studies - COLA. Grid Analysis and Display System (GrADS). IGES / COLA 4041 Powder Mill Road, Suite 30. Calverton, MD 20705-3106 USA, 2010 [Internet]. [cited 2010 Dec 20]. Available from: http://www.iges.org/grads/.

Centro de Previsão de Tempo e Estudos Climáticos - CPTEC. El Niño e La Niña. [cited 2013 July 21]. Available from: http://enos.cptec.inpe.br/.

Derrubadas. Prefeitura Municipal. Dados gerais [Internet]. [cited 2012 Dec 11]. Available from: http:// 
www.derrubadas-rs.com.br/siteantigo/index_arquivos/ page0001.htm.

Dwyer J, Schmidt G. The MODIS reprojection tool. In: Qu JJ, Gao W, Kafatos M, Murphy RE, Salomonson VV. Earth Science Satellite Remote Sensing. Berlin Heidelberg: Springer Berlin Heidelberg; 2006. p. 162177. http://dx.doi.org/10.1007/978-3-540-37294-3_9

Franchito SH, Rao VB, Vasques AC, Santo CME, Conforte JC. Validation of TRMM precipitation radar monthly rainfall estimates over Brazil. Journal of Geophysical Research 2009; 114: D02105 1-9. http:// dx.doi.org/10.1029/2007JD009580

Galvão LS, Breunig FM, Santos JR , Moura YM. Viewillumination effects on hyperspectral vegetation indices in the Amazonian tropical forest. International Journal of Applied Earth Observation and Geoinformation 2013; 21: 291-300. http://dx.doi.org/10.1016/j.jag.2012.07.005

Gunderson LH. Ecological resilience - in theory and application. Annual Review of Ecology and Systematics 2000; 31: 425-439. http://dx.doi.org/10.1016/j. jag.2012.07.005

Huete AR, Hua H, Qi J, Chehbouni A, Van Leeuwen WJD. Normalization of multidirectional red and NIR reflectances with the SAVI. Remote Sensing of Environment 1992; 41: 143-154. http://dx.doi. org/10.1016/0034-4257(92)90074-T

Huete A, Didan k, Miura T, Rodriguez EP, Gao X, Ferreira LG. Overview of the radiometric and biophysical performance of the MODIS vegetation indices. Remote Sensing of Environment 2002; 83(1-2): 195-213. http:// dx.doi.org/10.1016/S0034-4257(02)00096-2

Huntingford C, Zelazowski P, Galbraith D, Mercado LM, Sitch S, Fisher R, et al. Simulated resilience of tropical rainforests to $\mathrm{CO}_{2}$-induced climate change. Nature Geoscience 2013; 6: 268-273. http://dx.doi. org/10.1038/ngeo1741

Instituto Brasileiro de Geografia e Estatística - IBGE. Folhas SH 22 Porto Alegre e parte das folhas SH 21 e SI 22 Lagoa Mirim: Levantamento de Recursos Naturais. Geologia, geomorfologia, pedologia, vegetação, uso potencial da terra. Rio de Janeiro; 1986. v. 33.

Instituto Brasileiro de Geografia e Estatística - IBGE. Manual técnico da vegetação brasileira. 2. ed. Rio de Janeiro; 2012.

Irgang BE. A mata do alto Uruguai no RS. Ciência e Cultura 1980; (32): 323-324.

ITT Visual Information Solutions - ITT VIS. ENVI TMEnvironment for Visualizing for Images [Internet]. 4.5 ed. ${ }^{\circledR}$ ITT Visual Information Solutions; 2008. Available from: www.ittvis.com

Justice CO, Vermote E, Townshend JRG, DeFries R, Roy DP, Hall DK, et al. The Moderate Resolution Imaging Spectroradiometer (MODIS): Land Remote Sensing for Global Change Research. IEEE Transactions on Geoscience and Remote Sensing 1998; 36(4): 1228-1249. http://dx.doi.org/10.1109/36.701075

Justice CO, Townshend JRG, Vermote EF, Masuoka E, Wolfe RE, Saleous N, et al. An overview of MODIS Land data processing and product status. Remote Sensing of Environment 2002; 83(1-2): 3-15. http:// dx.doi.org/10.1016/S0034-4257(02)00084-6

Lee R, Yu F, Price KP. Evaluating vegetation phonological patterns in Inner Mongolia using NDVI timeseries analysis. International Journal of Remote Sensing 2002; 23(12): 2505-2512. http://dx.doi. org/10.1080/01431160110106087

Leite PF. Contribuição ao conhecimento fitoecológico do sul do Brasil. Ciência e Ambiente 2002; 24: 51-73.

Miura T, Huete AR, Yoshioka H, Holben BN. An error and sensitivity analysis of atmospheric resistant vegetation indices derived from dark target-based atmospheric correction. Remote Sensing of Environment 2001; 78(3): 284-298. http://dx.doi.org/10.1016/S00344257(01)00223-1

Parque Florestal Estadual do Turvo. Aspectos legais [Internet]. [cited 2012 Dec 13]. Available from: http:// www.derrubadas-rs.com.br/turvo.htm.

Passow MJ. TRMM - Tropical Rainfall Measuring Mission: Bringing remote sensing of precipitation into your classroom. Terrce Didatica 2010; 6(1): 03-08. [cited 2013 Jan 31]. Available from: http://ppegeo.igc.usp.br/ pdf/ted/v6n1/v6n1a02.pdf.

Phillips OL, Aragão LEOC, Lewis SL, Fisher JB, Lloyd J, López-González G, et al. Drought sensitivity of the Amazon rainforest. Science 2009; 323(5919): 1344-7. http://dx.doi.org/10.1126/science.1164033

Ponzoni FJ. Comportamento espectral da vegetação. In: Menezes PR, Madeira JS No. Sensoriamento Remoto: reflectância de alvos naturais. Brasília: UnB/Embrapa; 2001. 157-199.

Rio Grande do Sul. Secretaria Estadual do Meio Ambiente do Estado do Rio Grande do Sul (SEMA - RS). Plano de manejo do Parque Estadual do Turvo. Porto Alegre: Divisão de Unidades de Conservação do Estado do Rio Grande do Sul; 2005.

Rouse JW, Haas RH, Schell JA, Deering DW. Monitoring vegetation systems in the Great Plains with ERTS. In: Proceedings of the Earth Resources Technology Satellite-1 Symposium; 1973; Washington. Washington: NASA; 1973.

Santos AR, Peluzio TMO, Saito NS. Spring 5.1.2: passo a passo: Aplicações Práticas. Alegre: CAUFES; 2010. [cited 2012 Dec 10]. Available from: http://www. mundogeomatica.com.br/Livros/Livro_Spring_5.1.2_ Aplicacoes_Praticas/Livro_SPRING_512_ 
Pas soaPas so_Aplica\% C $3 \%$ A $7 \%$ C $3 \%$ A 3 o_ Pr\%C3\%Altica.pdf.

Silva L, Costa JF Fo, Lima VER, Almeida HA. Análise do albedo e do índice de vegetação da diferença normalizada (NDVI) em uma bacia hidrográfica, usando o SEBAL - Surface Energy Balance Algorithm for Land. In: Anais do XIII Simpósio Brasileiro de Geografia Física Aplicada; 2009; Viçosa. Viçosa: UFV; 2009. [cited 2012 Dec 15]. Available from: http://www. geo.ufv.br/simposio/simposio/trabalhos/trabalhos_ completos/eixo1/054.pdf.
Vibrans AC, Schramm VF. Perfil temporal de EVI/NDVI na bacia hidrográfica do rio Itajaí em Santa Catarina. In: Anais do Simpósio Brasileiro de Sensoriamento Remoto (SBSR); 1999; Natal. Natal: INPE; 2009. PMid:19943939 PMCid:PMC2789042.

Zhang X, Friedl MA, Schaaf CB, Strahler AH, Hodges JCF, Gao F, et al. Monitoring vegetation phenology using MODIS. Remote Sensing of Environment 2003; 84: 471475. http://dx.doi.org/10.1016/S0034-4257(02)00135-9 